\title{
ON AN EQUIVALENT DEFINITION OF FREE ENTROPY
}

\author{
Serban Teodor Belinschi
}

\begin{abstract}
In his second paper on free entropy, Voiculescu suggested five other possible definitions of the entropy of an $n$-tuple of selfadjoint random variables. We show that two of them are equivalent to the original one.
\end{abstract}

\section{Introduction}

Let $X_{1}, \ldots, X_{n}$ be $n$ selfadjoint random variables in the tracial $W^{*}$ - probability space $(\mathcal{M}, \tau)$. Denote by $\mathcal{M}_{k}^{s a}$ the space of $k \times k$ selfadjoint matrices over $\mathbb{C}$, let $\operatorname{Tr}_{k}$ be the (unnormalized) trace on $\mathcal{M}_{k}^{s a}$, and denote by $\tau_{k}$ the normalized trace, $\tau_{k}=\frac{1}{k} \operatorname{Tr}_{k}$. Then $\left(\mathcal{M}_{k}^{s a}\right)^{n}$ becomes a real Hilbert space with the norm

$$
\left\|\left(A_{1}, \ldots, A_{n}\right)\right\|_{2}=\left(\operatorname{Tr}_{k}\left(A_{1}^{2}+\cdots+A_{n}^{2}\right)\right)^{1 / 2} .
$$

Denote by $\lambda$ the Lebesgue measure corresponding to this norm (i.e. the Lebesgue measure normalized so that a cube with edges of length one has measure equal to one).

Let $\varepsilon>0, m>1, R>\max \left\{\left\|X_{1}\right\|, \ldots\left\|X_{n}\right\|\right\}$, and let $\Psi_{m, k}:\left(\mathcal{M}_{k}^{s a}\right)^{n} \rightarrow \mathbb{R}_{+}$ be defined by

$$
\Psi_{m, k}\left(A_{1}, \ldots, A_{n}\right)=\sum_{q=1}^{m} \sum_{1 \leq i_{1}, \ldots, i_{q} \leq n}\left|\tau_{k}\left(A_{i_{1}} \ldots A_{i_{q}}\right)-\tau\left(X_{i_{1}} \ldots X_{i_{q}}\right)\right| .
$$

Following Voiculescu's definition of microstate-based free entropy $\chi\left(X_{1}, \ldots, X_{n}\right)$, let

$$
\begin{aligned}
\Gamma_{R}\left(X_{1}, \ldots, X_{n} ; m, k, \varepsilon\right) & =\left\{\left(A_{1}, \ldots, A_{n}\right) \in\left(\mathcal{M}_{k}^{s a}\right)^{n}: \Psi_{m, k}\left(A_{1}, \ldots, A_{n}\right)<\varepsilon,\left\|A_{j}\right\| \leq R\right\} \\
\chi_{R}\left(X_{1}, \ldots, X_{n} ; m, k, \varepsilon\right) & =\log \lambda\left(\Gamma_{R}\left(X_{1}, \ldots, X_{n} ; m, k, \varepsilon\right)\right) \\
\chi_{R}\left(X_{1}, \ldots, X_{n} ; m, \varepsilon\right) & =\limsup _{k \rightarrow \infty}\left(k^{-2} \chi_{R}\left(X_{1}, \ldots, X_{n} ; m, k, \varepsilon\right)+2^{-1} n \log k\right) \\
\chi_{R}\left(X_{1}, \ldots, X_{n}\right) & =\lim _{\varepsilon \rightarrow 0, m \rightarrow \infty} \chi_{R}\left(X_{1}, \ldots, X_{n} ; m, \varepsilon\right) \\
\chi\left(X_{1}, \ldots, X_{n}\right) & =\sup _{R>0} \chi_{R}\left(X_{1}, \ldots, X_{n}\right) .
\end{aligned}
$$

Received November 19, 2002.

2000 Mathematics Subject Classification. Primary: 46L54; Secondary: 94A17. 
In Section 7.1 of [3], two alternative quantities were defined by Voiculescu as follows. Let $\mathcal{D}_{1, R}\left(X_{1}, \ldots, X_{n} ; m, k, \varepsilon\right)$ be the set of probability densities $f$ : $\left(\mathcal{M}_{k}^{s a}\right)^{n} \rightarrow \mathbb{R}_{+}$(i.e. $\left.\int f d \lambda=1\right)$ so that $\int f\left(A_{1}, \ldots, A_{n}\right) \Psi_{m, k}\left(A_{1}, \ldots, A_{n}\right) d \lambda \leq \varepsilon$ and $f\left(A_{1}, \ldots, A_{n}\right)=0$ unless $\left\|A_{j}\right\| \leq R(1 \leq j \leq n)$. Then

$\chi_{R}^{(1)}\left(X_{1}, \ldots, X_{n} ; m, k, \varepsilon\right)=\sup \left\{-\int f \log f d \lambda: f \in \mathcal{D}_{1, R}\left(X_{1}, \ldots, X_{n} ; m, k, \varepsilon\right)\right\}$.

$\chi^{(1)}$ is now defined following the same steps as in the definition of $\chi$. One can also define $\chi_{\infty}$ and $\chi_{\infty}^{(1)}$ by setting $R=\infty$ from the beginning, that is, by leaving out the conditions on the norms of the $A_{j}$ 's. Since the characteristic function of $\Gamma_{R}\left(X_{1}, \ldots, X_{n} ; m, k, \varepsilon\right)$ (properly normalized) belongs to $\mathcal{D}_{1, R}\left(X_{1}, \ldots, X_{n} ; m, k, \varepsilon\right)$, we conclude that

$$
\chi_{R}^{(1)}\left(X_{1}, \ldots, X_{n} ; m, k, \varepsilon\right) \geq \chi_{R}\left(X_{1}, \ldots, X_{n} ; m, k, \varepsilon\right) .
$$

It follows that $\chi \leq \chi^{(1)}$, and, likewise, $\chi_{\infty} \leq \chi_{\infty}^{(1)}$.

It has already been proved in [1] that $\chi_{\infty}=\chi$. In this paper we shall prove that $\chi_{\infty}^{(1)}=\chi_{\infty}$, which will obviously imply that $\chi_{\infty}^{(1)}=\chi^{(1)}=\chi=\chi_{\infty}$. As an immediate consequence, we obtain a new way to define free entropy replacing $\chi_{R}\left(X_{1}, \ldots, X_{n} ; m, k, \varepsilon\right)$ by:

$$
\chi_{\infty}^{(1)}\left(X_{1}, \ldots, X_{n} ; m, k, \varepsilon\right)=\log \int e^{-s\left(\Psi_{m, k}\left(A_{1}, \ldots, A_{n}\right)-\varepsilon\right)} d \lambda,
$$

and continuing as in the original definition. Here $s$ is an appropriate positive number depending on $X_{1}, X_{2}, \ldots, X_{n}, m, \varepsilon$ and $k$.

\section{The main result}

Given a probability density $f: \mathbb{R}^{p} \rightarrow[0,+\infty)$, i.e. $\int_{\mathbb{R}^{p}} f(x) d \lambda(x)=1$, we denote by $I(f)=-\int_{\mathbb{R}^{p}} f(x) \log f(x) d \lambda(x)$ the classical entropy of $f$. A density of special interest is the Gaussian density $G_{a^{2}}^{(p)}$ defined on $\mathbb{R}^{p}$ by $G_{a^{2}}^{(p)}(x)=$ $\left(\frac{1}{2 \pi a^{2}}\right)^{\frac{p}{2}} e^{-\frac{\|x\|_{2}^{2}}{2 a^{2}}}$.

We will need the following extension of Shannon's classical inequality (see [2], pp. 55-57).

Lemma 2.1. Let $\Omega \subset \mathbb{R}^{p}$ be a set of positive Lebesgue measure, and $\varepsilon>0$ be fixed. If $\varphi$ is a positive continuous function on $\Omega$ such that $\int_{\Omega} \varphi \geq \varepsilon \lambda(\Omega)$, $\varphi^{-1}([0, \varepsilon))$ is nonempty, and both $e^{-\varphi}$ and $\varphi^{2} e^{-\varphi}$ are integrable on $\Omega$, then there is a number $s \in(0, \infty)$ depending on $\varphi, p$, and $\varepsilon$ such that $M:=$

$\max \left\{I(f): f \geq 0, f=0\right.$ on $\left.\mathbb{R}^{n} \backslash \Omega, \int_{\Omega} f(x) d \lambda(x)=1, \int_{\Omega} f(x) \varphi(x) d \lambda(x)=\varepsilon\right\}$

is attained for $f=\left(\int_{\Omega} e^{-s \varphi(x)} d \lambda(x)\right)^{-1} e^{-s \varphi}$ and equals $\log \int_{\Omega} e^{-s(\varphi(x)-\varepsilon)} d \lambda$. 
Proof. For each $s>0$, let $f_{s}(x)=\left(\int_{\Omega} e^{-s \varphi(y)} d \lambda(y)\right)^{-1} e^{-s \varphi(x)}$. Consider the function $h:(0, \infty) \rightarrow(0, \infty)$ given by $h(s)=\int_{\Omega} \varphi(x) f_{s}(x) d \lambda(x)$. Since both $e^{-\varphi}$ and $\varphi^{2} e^{-\varphi}$ are integrable on $\Omega$, we can differentiate under the integral sign, and a straightforward calculation shows that

$$
h^{\prime}(s)=-\int_{\Omega}\left(\varphi(x)-\int_{\Omega} \varphi(y) f_{s}(y) d \lambda(y)\right)^{2} f_{s}(x) d \lambda(x),
$$

so $h^{\prime}(s)<0$, We also have that

$$
\lim _{s \rightarrow \infty} h(s)=\min _{x \in \Omega} \varphi(x),
$$

which is strictly less than $\varepsilon$, and $\lim _{s \rightarrow 0} h(s)$ equals $\frac{1}{\lambda(\Omega)} \int_{\Omega} \varphi$ if $\lambda(\Omega)<\infty$ and infinity if $\lambda(\Omega)=\infty$, which are both greater than or equal to $\varepsilon$. Hence the existence of $s$ is assured by the monotonicity of $h$. Moreover, since $\varphi$ is nonconstant, $s$ is unique with this property.

Let

$$
g \in\left\{f: f \geq 0, \int_{\Omega} f d \lambda=1, \int_{\Omega} f \varphi d \lambda=\varepsilon\right\} .
$$

We may assume that $g(x)<\infty$ for all $x \in \Omega$. If $I(g)=-\infty$, then it is obvious that $I\left(f_{s}\right)>I(g)$. So suppose that $I(g)>-\infty$. Consider the function $\eta$ : $[0,1] \rightarrow \mathbb{R}$ defined by $\eta(t)=I\left((1-t) f_{0}+t g\right)$. This function is continuous on $[0,1]$, differentiable on $[0,1)$, and twice differentiable on $(0,1)$. Moreover, $\eta^{\prime}(t)=-\int_{\Omega}\left(g-f_{s}\right)\left(\log \left((1-t) f_{s}+t g\right)+1\right)$, and $\eta^{\prime \prime}(t)=-\int_{\Omega} \frac{\left(g-f_{s}\right)^{2}}{(1-t) f_{s}+t g}$. (This follows immediatly from the dominated convergence theorem. Indeed,

$$
\left|\left(g-f_{s}\right)\left(\log \left((1-t) f_{s}+t g\right)+1\right)\right| \leq g+f_{s}+\left|\left(g-f_{s}\right) \log \left((1-t) f_{s}+t g\right)\right|
$$

for all $t \in[0,1)$, and for any compact interval $K \subset[0,1), \sup _{t \in K} \mid\left(g-f_{s}\right) \log ((1-$ $\left.t) f_{s}+t g\right) \mid$ is integrable on $\Omega$. Also,

$$
\left|\frac{\left(g-f_{s}\right)^{2}}{(1-t) f_{s}+t g}\right| \leq \frac{1}{t} g+\frac{1}{1-t} f_{s}
$$

for all $t \in(0,1)$, and, of course, $\sup _{t \in K}\left|\frac{1}{t} g+\frac{1}{1-t} f_{s}\right|$ is integrable on $\Omega$ for any compact interval $K \subset(0,1)$.)

By direct computation, we obtain that $\eta^{\prime}(0)=0$. Also, $\eta^{\prime}$ is continuous on $[0,1)$, and $\eta^{\prime \prime}(t) \leq 0$ for all $t \in(0,1)$, so the point $t=0$ is a global maximum for $\eta$. This proves that $I\left(f_{s}\right)=M$ and concludes the proof.

Remark 2.2. To see how this lemma extends Shannon's inequality, set $\Omega=\mathbb{R}^{n}$, and $\varphi(x)=\|x\|_{2}^{2}$. In this case the extremal density $f$ is $G_{\varepsilon}^{(p)}$ and $I\left(G_{\varepsilon}^{(p)}\right)=$ $2^{-1} p \log \left(2 \pi e p^{-1} \varepsilon\right)$. Also note that for $\lambda(\Omega)<+\infty$ and $\varphi=\varepsilon$, the extremal density is $\frac{1}{\lambda(\Omega)} \chi_{\Omega}$ and $I\left(\frac{1}{\lambda(\Omega)} \chi_{\Omega}\right)=\log \lambda(\Omega)$.

Now we can prove the main result of the paper. 
Theorem 2.1. Let $X_{1}, \ldots, X_{n}$ be selfadjoint random variables in a tracial $W^{*}$ probability space $(\mathcal{M}, \tau)$. Then

$$
\chi_{\infty}\left(X_{1}, \ldots, X_{n}\right)=\chi_{\infty}^{(1)}\left(X_{1}, \ldots, X_{n}\right) .
$$

Proof. As noted in the introduction, $\chi_{\infty}\left(X_{1}, \ldots, X_{n}\right) \leq \chi_{\infty}^{(1)}\left(X_{1}, \ldots, X_{n}\right)$, so it is enough to prove the opposite inequality. Let $m \geq 2$ and $\varepsilon \in(0,1 / 2)$ be fixed. Let $V_{0}=\left\{\left(A_{1}, \ldots, A_{n}\right) \in\left(\mathcal{M}_{k}^{s a}\right)^{n}: \Psi_{m, k}\left(A_{1}, \ldots, A_{n}\right) \leq \varepsilon\right\}$, and $V_{1}=\left(\mathcal{M}_{k}^{s a}\right)^{n} \backslash V_{0}$. Consider $f_{0} \in \mathcal{D}_{1, \infty}\left(X_{1}, \ldots, X_{n} ; m, k, \varepsilon^{2}\right)$ such that $I\left(f_{0}\right)=$ $\max \left\{I(f): f \in \Gamma_{\infty}^{(1)}\left(X_{1}, \ldots, X_{n} ; m, k, \varepsilon^{2}\right)\right\}$, and define $f_{0}^{(i)}=c_{i}{ }^{-1} f_{0} \chi_{V_{i}}$, where $c_{i}=\int_{V_{i}} f, i=0,1$ depend on $X_{1}, \ldots, X_{n}, m, k$ and $\varepsilon$. Then we have:

$$
\varepsilon^{2} \geq \int f_{0} \Psi_{m, k} \geq \int_{V_{1}} f_{0} \Psi_{m, k} \geq \varepsilon \int_{V_{1}} f_{0}=\varepsilon c_{1},
$$

so $c_{1} \leq \varepsilon$, and $c_{0} \geq 1-\varepsilon$.

Since $f_{0} \in \mathcal{D}_{1, \infty}\left(X_{1}, \ldots, X_{n} ; m, k, \varepsilon^{2}\right)$,

$$
\int f_{0}^{(1)} \Psi_{m, k}=\left(\int_{V_{1}} f_{0}\right)^{-1} \int_{V_{1}} f_{0} \Psi_{m, k}<\frac{\varepsilon^{2}}{c_{1}},
$$

which implies that $f_{0}^{(1)} \in \mathcal{D}_{1, \infty}\left(X_{1}, \ldots, X_{n} ; m, k, \frac{\varepsilon^{2}}{c_{1}}\right)$.

Now,

$$
\begin{aligned}
I\left(f_{0}\right)= & c_{0} I\left(f_{0}^{(0)}\right)+c_{1} I\left(f_{0}^{(1)}\right)-c_{0} \log c_{0}-c_{1} \log c_{1} \\
\leq & c_{0} I\left(\frac{1}{\left|V_{0}\right|} \chi_{V_{0}}\right)+c_{1} I\left(G_{k\left(C^{2}+n \varepsilon^{2} / c_{1}\right)}^{\left(n k^{2}\right)}-c_{0} \log c_{0}-c_{1} \log c_{1}\right. \\
= & c_{0} \chi_{\infty}\left(X_{1}, \ldots, X_{n} ; m, k, \varepsilon\right)+c_{1} \frac{n k^{2}}{2}\left(\log \frac{2 \pi e\left(C^{2}+n \varepsilon^{2} / c_{1}\right)}{n}-\log k\right) \\
& -c_{0} \log c_{0}-c_{1} \log c_{1},
\end{aligned}
$$

where $C^{2}=\tau\left(X_{1}{ }^{2}+\cdots+X_{n}{ }^{2}\right)$. Here we used Lemma 2.1 in the last inequality.

Dividing by $k^{2}$ and adding $\frac{n}{2} \log k$ in (1), we obtain:

$$
\begin{aligned}
\frac{1}{k^{2}} I\left(f_{0}\right)+\frac{n}{2} \log k \leq & c_{0}\left(\frac{1}{k^{2}} \chi_{\infty}\left(X_{1}, \ldots, X_{n} ; m, k, \varepsilon\right)+\frac{n}{2} \log k\right) \\
& +c_{1} \frac{n}{2} \log \frac{2 \pi e\left(C^{2}+n \varepsilon^{2} / c_{1}\right)}{n}-\frac{1}{k^{2}}\left(c_{0} \log c_{0}+c_{1} \log c_{1}\right) .
\end{aligned}
$$

As $k \rightarrow \infty$, this yields

$$
\begin{gathered}
\chi_{\infty}^{(1)}\left(X_{1}, \ldots, X_{n} ; m, \varepsilon^{2}\right) \leq \limsup _{k \rightarrow \infty} c_{0}\left(\frac{1}{k^{2}} \chi_{\infty}\left(X_{1}, \ldots, X_{n} ; m, k, \varepsilon\right)+\frac{n}{2} \log k\right) \\
+\limsup _{k \rightarrow \infty} c_{1} \frac{n}{2} \log \frac{2 \pi e\left(C^{2}+n \varepsilon^{2} / c_{1}\right)}{n}
\end{gathered}
$$


Since $\lim _{t \rightarrow 0, t>0} t \log \frac{2 \pi e\left(C^{2}+n \varepsilon^{2} / t\right)}{n}=0$, the function

$$
s(\varepsilon):=\sup \left\{t \frac{n}{2} \log \frac{2 \pi e\left(C^{2}+n \varepsilon^{2} / t\right)}{n}: t \in(0, \varepsilon]\right\}
$$

does not depend on $k$ and $m$ and tends to zero as $\varepsilon \rightarrow 0$.

Hence

$\chi_{\infty}^{(1)}\left(X_{1}, \ldots, X_{n} ; m, \varepsilon^{2}\right) \leq \limsup _{k \rightarrow \infty} c_{0}\left(\frac{1}{k^{2}} \chi_{\infty}\left(X_{1}, \ldots, X_{n} ; m, k, \varepsilon\right)+\frac{n}{2} \log k\right)+s(\varepsilon)$

On the other hand, since $1 \geq c_{0} \geq 1-\varepsilon$, we have that

$$
\begin{aligned}
& \limsup _{k \rightarrow \infty} c_{0}\left(\frac{1}{k^{2}} \chi_{\infty}\left(X_{1}, \ldots, X_{n} ; m, k, \varepsilon\right)+\frac{n}{2} \log k\right) \leq \\
& \quad \max \left\{(1-\varepsilon) \chi_{\infty}\left(X_{1}, \ldots, X_{n} ; m, \varepsilon\right), \chi_{\infty}\left(X_{1}, \ldots, X_{n} ; m, \varepsilon\right)\right\} .
\end{aligned}
$$

This allows us to conclude that

$$
\begin{aligned}
& \chi_{\infty}^{(1)}\left(X_{1}, \ldots, X_{n} ; m, \varepsilon^{2}\right) \leq \\
& \quad \max \left\{(1-\varepsilon) \chi_{\infty}\left(X_{1}, \ldots, X_{n} ; m, \varepsilon\right), \chi_{\infty}\left(X_{1}, \ldots, X_{n} ; m, \varepsilon\right)\right\}+s(\varepsilon) .
\end{aligned}
$$

Taking limits when $m \rightarrow \infty$ and $\varepsilon \rightarrow 0$ in the previous inequality, we obtain

$$
\chi_{\infty}^{(1)}\left(X_{1}, \ldots, X_{n}\right)=\chi_{\infty}\left(X_{1}, \ldots, X_{n}\right) .
$$

\section{Acknowledgement}

I wish to thank my advisor Professor Hari Bercovici for the constant help that made this paper possible.

\section{References}

[1] S. T. Belinschi, H. Bercovici, A property of free entropy, to appear in Pacific J. Math.

[2] C. E. Shannon, The Mathematical Theory of Communication, The University of Illinois Press, Urbana, IL, 1949.

[3] D. Voiculescu, The analogues of entropy and of Fisher's information measure in free probability theory. II, Invent. Math. 118 (1994), 411-440.

Mathematics Department, Indiana University, Bloomington, Indiana 47405, U.S.A. E-mail address: sbelinsc@indiana.edu

Institute of Mathematics, Romanian Academy, P.O. Box 1-764, Bucharest, RO70700, ROMANIA. 